\title{
COMPOSITION, PROPERTY AND STRUCTURE OF CHROME COMPLEXES IN NEUTRALIZING EFFLUENT
}

\author{
YANPING GAO ${ }^{1,2}$, JUN HU ${ }^{1}$, JIN WEI ZHANG ${ }^{1}$, WUYONG CHEN ${ }^{1,2^{*}}$ \\ ${ }^{1}$ Sichuan University, Key Laboratory of Leather Chemistry and Engineering of the Ministry of \\ Education, 610065, P. R. China,wuyong.chen@163.com \\ ${ }^{2}$ Sichuan University, National Engineering Laboratory for Clean Technology of Leather \\ Manufacture, Chengdu 610065, P. R. China
}

\begin{abstract}
In order to interpret the reason of chromium complexes release from the chrome tanned leather during the neutralizing process, the hide powder was tanned by chromium sulfate liquor with $33 \%$ basicity (BCS), and then the tanned collagen was treated by general neutralizing process to obtain neutralizing effluent. Ion exchange chromatography (IEC) and gel filtration chromatography (GFC) were used to separate neutralizing effluent; Ultraviolet-visible absorption spectroscopy (UV-Vis) and Fourier Transform Infrared Spectroscopy (FT-IR) were combined to analyze the structure of chrome complexes in the neutralizing effluent. The results indicated that the neutralizing effluent mainly consisted of anionic-zero valent, monovalent and bivalent chrome complexes; the molecular weight of chrome complexes in neutralizing effluent was far less than BCS; releasing chromium complexes were mainly combined with ligands through single point coordination and $\mathrm{Cr}^{3+}$ was mainly combined with $\mathrm{SO}_{4}{ }^{2-}$ and $\mathrm{HCOO}^{-}$by single point coordinate bond. Combined all of the results above, it could deduce that the main constitutes of anionic-zero valent and low electro-positive, smaller molecular, mainly combined with single point coordination are the properties and structural characteristics of the chromium complexes released from chrome tanned leather in neutralizing process.
\end{abstract}

Keywords: hide powder; neutralizing effluent; chrome complex

\section{INTRODUCTION}

Chrome-tanned leather has excellent performance, so chrome tanning is still dominant in the leather industry. But the chromium pollution problem is increasingly brought to the attention, chrome tanning method is facing severe challenges, therefore minimize chromium pollution has significant environmental benefits. After chrome tanning, the chrome release in post-tanning process in the post-tanning process like rewetting, neutralizing, retanning, dyeing and fatliquoring effluents, leading $\mathrm{Cr}_{2} \mathrm{O}_{3}$ content of post-tanning effluents reached $10 \sim 450 \mathrm{mg} / \mathrm{L}$ that is significantly more than the limit standard (Zhou et al., 2012) are still needed to face. In order to solve chrome pollution problems, chrome release in post-tanning process must be highly valued and adopt corresponding measures.

Organic retanning agents, dyestuffs and fatliquoring agents are mainly anionic materials used in subsequent process of wet blue. The neutralizing process is usually used to raise the $\mathrm{pH}$ and adjust the charge to promote penetration of anionic materials. Therefore, there is a certain amount of chrome released in neutralizing process. This article studied the composition, structure and properties of chrome complexes released from the chrome tanned skin collagen in neutralizing effluent.

The hide powder was tanned by chromium sulfate liquor with 33\% basicity (BCS), and then the tanned collagen was treated by general neutralizing process to obtain neutralizing effluent. Ion exchange chromatography (IEC) and gel filtration chromatography (GFC) were used to separate neutralizing effluent; Ultraviolet-visible absorption spectroscopy (UV-Vis) and Fourier Transform Infrared Spectroscopy (FTIR) were used to analyze the structure of chrome complexes. 


\section{EXPERIMENTAL}

\section{Preparation of Neutralizing Effluent}

Hide powder was tanned by $0.2 \mathrm{~mol} / \mathrm{L} \mathrm{BCS}$ for $2 \mathrm{~h}$ at constant temperature bath oscillator $\left(25^{\circ} \mathrm{C}, 200 \mathrm{r} / \mathrm{min}\right) .1 .2 \% \mathrm{NaHCO}_{3}$ was added four times to basify, the final $\mathrm{pH}$ of tanning liquor was 3.8. The tanned hide powder was dried at $40^{\circ} \mathrm{C}$ after tanning.

$0.2 \%$ formic acid and $150 \%$ water were added in dried hide powder, and then put in constant temperature bath oscillator for $2 \mathrm{~h}\left(38^{\circ} \mathrm{C}, 200 \mathrm{r} / \mathrm{min}\right)$. Then, the hide powder was washed by running water for $10 \mathrm{~min} .2 \%$ sodium formate and $150 \%$ water were added and put in constant temperature bath oscillator for $90 \mathrm{~min}\left(35^{\circ} \mathrm{C}, 200 \mathrm{r} / \mathrm{min}\right)$. After filtered with nylon filter cloth, neutralizing effluent was prepared.

\section{Ion Exchange Chromatography Separation}

$5 \mathrm{ml}$ neutralizing effluent was filtered by $450 \mathrm{~nm}$ microporous membranes, and then added into SP Sephadex C-25 column (inner diameter $2.0 \mathrm{~cm}$, packed $20.0 \mathrm{~cm}$ ). Elution conditions were as following: eluent velocity for $2.5 \mathrm{~mL} / \mathrm{min}$, followed by using $\mathrm{H}_{2} \mathrm{O}$, $\mathrm{NaClO}_{4}(0.5,1.0,2.0 \mathrm{~mol} / \mathrm{L}), \mathrm{HCl}(2.0,3.0 \mathrm{~mol} / \mathrm{L})$ to elute. UV-Vis spectrophotometer (Shanghai Jinghua Technology Corporation) was used to detect absorbance at $420 \mathrm{~nm}$ and compare with BCS separated compositions (Li et al., 1992).

\section{Gel Filtration Chromatography Separation}

$5 \mathrm{ml}$ neutralizing effluent was filtered by $450 \mathrm{~nm}$ microporous membranes, and then added into SP Sephadex G-25 column (inner diameter $2.0 \mathrm{~cm}$, packed $80.0 \mathrm{~cm}$ ). Elution conditions were as following: eluent velocity for $2.5 \mathrm{~mL} / \mathrm{min}$ and $\mathrm{H}_{2} \mathrm{O}$ was used to elute. UV-Vis spectrophotometer was used to detect absorbance at $420 \mathrm{~nm}$ and compare with BCS separated compositions (Davis and Scroggie, 1973).

\section{UV-Vis Analysis}

After IEC separation, collected components were freeze-dried and then dissolved by distilled water. UV-Vis spectrophotometer was used to scan obtained liquor at 300-800 $\mathrm{nm}$. R was calculated by molar absorption coefficient of chrome in $420 \mathrm{~nm}$ and $580 \mathrm{~nm}$.

\section{FT-IR Analysis}

FT-IR spectrum in the $4000-400 \mathrm{~cm}^{-1}$ was recorded with a Nicolet 10 FT-IR spectrophotometer (American Thermo Scientific Corporation). Samples were prepared by grinding about $1 \mathrm{mg}$ freeze-dried components with $100 \mathrm{mg} \mathrm{KBr}$, and the mixture was pressed into very thin disks and scanned for 32 times. 
ICAMS $2016-6^{\text {th }}$ International Conference on Advanced Materials and Systems

\section{RESULTS AND DISCUSSIONS}

\section{Charge Characteristics of Chrome Complexes in Neutralizing Effluent}

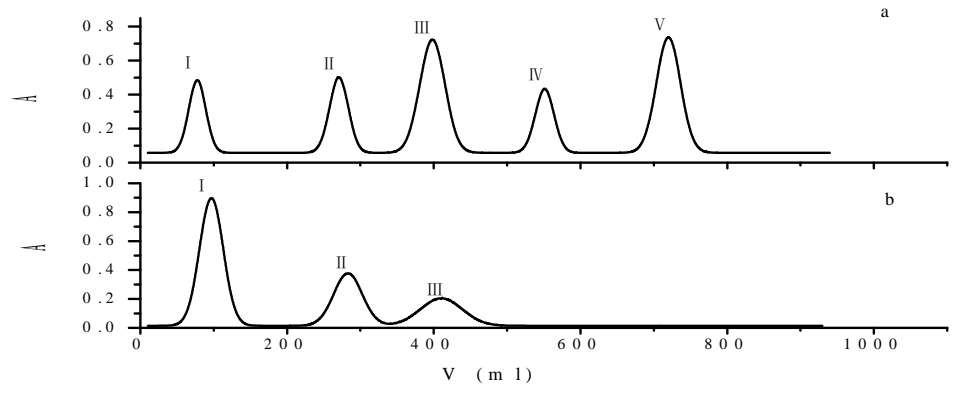

Figure 1. Ion exchange chromatography of BCS (a) and neutralizing effluent (b)

IEC of BCS and neutralizing effluent are shown in Fig.1. As can be seen from Fig.1, at the same elution conditions, BCS is separated into five components (anionic-zero valent, monovalent, bivalent, trivalent and tetravalent chrome complexes), and neutralizing effluent is divided into three components (anionic-zero valent, monovalent and bivalent chrome complexes). Based on IEC peak area, the composition of chrome complexes in BCS and neutralizing effluent can be calculated and listed in Table 1.

\section{Molecular Weight of Chrome Complexes in Neutralizing Effluent}

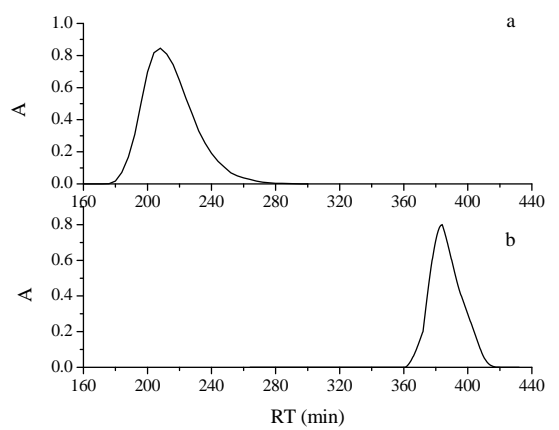

Figure 2. Gel filtration chromatography of BCS (a) and neutralizing effluent (b)

GFC is often used to analyze the molecular weight distribution to study the structure of coordination compounds. The retention time of the large molecular weight complexes is shorter while the small molecular weight is longer during the GFC separation process. GFC results of BCS and neutralizing effluent are shown in Fig.2. As shown in Fig.2, during $172 \mathrm{~min}$ and $280 \mathrm{~min}$, chrome complexes in BCS are washed off completely (Fig.2 a); between $368 \mathrm{~min}$ and $408 \mathrm{~min}$, chrome complexes in neutralizing effluent are washed off completely (Fig.2 b). Accordingly, the molecular weight of chrome complexes in neutralizing effluent is far less than BCS. The small molecular weight 
Composition, Property and Structure of Chrome Complexes in Neutralizing Effluent

chrome complexes cannot combine with skin collagen by stable combination, therefore small chrome complexes are easier to release.

\section{UV-Vis Results}

Table 2. $\mathrm{R}$ values of each constituent in neutralizing effluent

\begin{tabular}{cccccc}
\hline R values & I & II & III & IV & V \\
\hline BCS & 1.08 & 1.10 & 1.09 & 1.15 & 1.36 \\
neutralizing effluent & 0.95 & 0.80 & 0.83 & - & - \\
\hline
\end{tabular}

The combination way between ligands and $\mathrm{Cr}^{3+}$ can be determined by $\mathrm{R}$ values, $\mathrm{R}$ values of each constituent in neutralizing effluent are listed in Table 2. The results in Table 2 showed that, the R value of chrome complex components I, II, III, IV and V in $\mathrm{BCS}$ are $1.08,1.10,1.09,1.15$ and 1.36 respectively, however, the $\mathrm{R}$ value of chrome complex components I, II and III in neutralizing effluent are $0.95,0.80$ and 0.83 . The $\mathrm{R}$ values of I, II and III are all less than 1.19 indicating that $\mathrm{Cr}^{3+}$ mainly combined with ligands through single point coordination and there are less bridge linkages between $\mathrm{Cr}^{3+}$ and ligands.

\section{FT-IR Results of Chrome Complex in Neutralizing Effluent}

Characteristic absorption peak and coordination mode of I to III components of chrome complex in neutralizing effluent are shown in Table 3. It is seen that 4 absorption peaks are found in $900-1300 \mathrm{~cm}^{-1}$ for chromium complex in component I, which indicate that interconnection mode for $\mathrm{SO}_{4}{ }^{2-}$ and central ion $\mathrm{Cr}^{3+}$ is two-point coordination. In the same way, 2 and 3 absorption peaks in component II and III respectively demonstrate that $\mathrm{SO}_{4}{ }^{2-}$ is free state and coordinate with $\mathrm{Cr}^{3+}$ by single point coordination.

One asymmetric and one symmetric stretching vibration absorption peak are respectively found in $1550-1680 \mathrm{~cm}^{-1}$ and $1370-1465 \mathrm{~cm}^{-1}$ of chrome complex in component I, II and III, moreover, the interval of these two absorption peaks are small. Therefore single point coordination is speculated between $\mathrm{Cr}^{3+}$ and $\mathrm{HCOO}^{-}(\mathrm{Chen}$ and $\mathrm{Li}, 2011)$. 
ICAMS $2016-6^{\text {th }}$ International Conference on Advanced Materials and Systems

Table 3. FT-IR absorption peak and coordination mode of chromium complex in neutralizing effluent

\begin{tabular}{|c|c|c|c|c|c|}
\hline Component & $\begin{array}{l}\text { Scope } \\
\left(\mathrm{cm}^{-1}\right)\end{array}$ & Peak $\left(\mathrm{cm}^{-1}\right)$ & Num. & Ligand & $\begin{array}{c}\text { Coordination } \\
\text { mode }\end{array}$ \\
\hline \multirow{4}{*}{ I } & $900-1300$ & $995,1048,1148,1114$ & 4 & $\mathrm{SO}_{4}{ }^{2-}$ & two-point \\
\hline & $1680-1550$ & 1633 & \multirow[b]{2}{*}{2} & \multirow{2}{*}{$\mathrm{HCOO}^{-}$} & \multirow{2}{*}{ single point } \\
\hline & $1465-1370$ & 1405 & & & \\
\hline & 3400 & 3439 & 1 & $\mathrm{OH}$ & ---- \\
\hline \multirow{4}{*}{ II } & $900-1300$ & 941,1088 & 2 & $\mathrm{SO}_{4}{ }^{2-}$ & free \\
\hline & $1680-1550$ & 1630 & \multirow{2}{*}{1} & \multirow{2}{*}{$\mathrm{HCOO}^{-}$} & \multirow{2}{*}{ single point } \\
\hline & $1465-1370$ & 1430 & & & \\
\hline & 3400 & 3423 & 1 & $\mathrm{OH}$ & ---- \\
\hline \multirow{4}{*}{ III } & $900-1300$ & $941,1087,1141$ & 3 & $\mathrm{SO}_{4}{ }^{2-}$ & single point \\
\hline & $1680-1550$ & 1631 & \multirow{2}{*}{1} & \multirow{2}{*}{$\mathrm{HCOO}^{-}$} & \multirow{2}{*}{ single point } \\
\hline & $1465-1370$ & 1422 & & & \\
\hline & 3400 & 3441 & 1 & $\mathrm{OH}$ & ---- \\
\hline
\end{tabular}

Chromium complex Structure in neutralizing effluent is speculated by valence state of IEC (Tab.1) and R values (Tab.2). Its possible structures are shown in Fig.3.

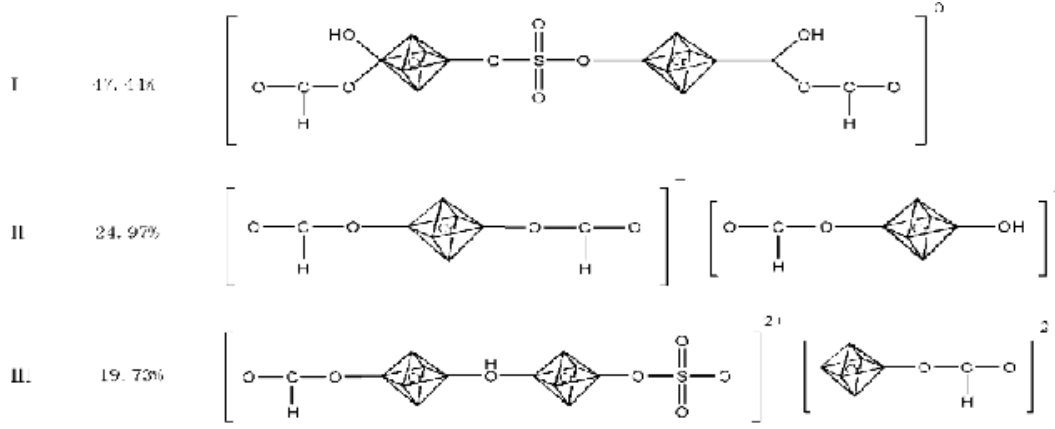

Figure 3. Possible structures of chrome complexes in neutralizing effluent

\section{CONCLUSIONS}

The results of IEC and GFC indicate that the neutralizing effluent mainly consisted of anionic-zero valent and low-positive electricity $(+1$ and +2$)$ chrome complexes; the molecular weight of chrome complexes in neutralizing effluent is far less than the chromium sulfate liquor with $33 \%$ basicity. The results of UV-Vis and FT-IR show that releasing chromium complexes is mainly combined with $\mathrm{SO}_{4}{ }^{2-}$ and $\mathrm{HCOO}^{-}$by single point coordinate bond. Combined all of the results above, the main characteristics of the chromium complexes released from neutralizing process of chrome tanned leather are anionic-zero valent and low electro-positive component, smaller molecular, mainly combined with ligands through single point coordination.

\section{Acknowledgements}

This work is financially supported by the National Natural Science Foundation of China (No. 21576171). 
Composition, Property and Structure of Chrome Complexes in Neutralizing Effluent

\section{REFERENCES}

Chen, W.Y. and Li, G.Y. (2011), Tanning Chemistry, China Light Industry Press (the third edition), Beijing. Davis, M.H. and Scroggie, J.G. (1973), "Investigation of Commercial Chrome-Tanning Systems, Part I", Journal of the Society of Leather Technologists and Chemists, 57(1), 13-18.

Li, G.Y., Cheng, J. and Zhang, M.R. (1992), "The research on the composition of chromium sulfate tanning liquor", China Leather, 21(10), 8-19.

Zhou, J., Hu, S.X., Wang, Y.N., He, Q., Liao, X.P., Zhang, W.H. and Shi, B. (2012), "Release of Chrome Tanning and Post Tanning Process", Journal of the Society of Leather Technologists and Chemists, 96(4), $157-161$. 